\title{
Escala de Bienestar Psicológico para Adolescentes
}

\section{Psychological Well-Being Scale for Adolescents}

\author{
Marcela B. González-Fuentes ${ }^{1}$ y Patricia Andrade Palos ${ }^{2}$
}

\section{Resumen}

El propósito de este trabajo fue diseñar y validar una escala para evaluar la percepción de Bienestar Psicológico (BP) en adolescentes mexicanos. La fase I fue exploratoria, se realizaron dos estudios, uno para identificar el significado que los adolescentes le dieron al BP, y otro para diseñar y validar la escala. La fase II permitió confirmar las características psicométricas. En el primero participaron 30 adolescentes de 15 a 20 años; y en el segundo 665, de 14 a 20 años $(M=16.7$; $\mathrm{DE}=1.36)$. En la fase II participaron 1064 estudiantes de 15 a 20 años $(M=16.10 ; D E=.941)$. Los resultados agruparon 29 reactivos en siete factores $(\alpha=.76)$, que explicaron el $60.51 \%$ de la varianza total: crecimiento personal $(\alpha=.79)$, relaciones positivas con otros $(\alpha=.81)$, propósito de vida $(\alpha=.80)$, auto-aceptación $(\alpha=.74)$, planes a futuro $(\alpha=.77)$, rechazo personal $(\alpha=.77)$ y control personal $(\alpha=.83)$.

Palabras clave: bienestar psicológico, adolescentes, funcionamiento positivo, auto-realización

\begin{abstract}
The purpose was to design and validate a scale to assess the perception of Psychological Well-Being (PWB) in Mexican adolescents. Phase I was exploratory and was composed of two studies, one to identify the meaning given by adolescents to PWB, and the other to design and validate it. Psychometric characteristics were confirmed in Phase II. The first study involved 30 teenagers, the second 665, from 14 to 20 years $(\mathrm{M}=16.7 ; \mathrm{SD}=1.36)$. In Phase II 1064 students between 15 and 20 years participated $(\mathrm{M}=16.10 ; \mathrm{SD}=.941)$. Results grouped 29 items in seven factors $(\alpha=.76)$, which explained the $60.51 \%$ of total variance: personal growth $(\alpha=.79)$, positive relationships with others $(\alpha=.81)$, purpose of life $(\alpha=.80)$, self-acceptance $(\alpha=.74)$, plans for the future $(\alpha=.77)$, personal rejection $(\alpha=.77)$ and personal control $(\alpha=.83)$.
\end{abstract}

Keywords: psychological well-being, adolescents, positive functioning performance, self-realization

\footnotetext{
${ }^{1}$ Doctora en Psicología. Facultad de Psicología, Universidad Nacional Autónoma de México. Profesora Titular Tiempo completo. Ave. Universidad 3004, Col. Copilco-Universidad, CP. 04510, Delegación Coyoacán, México, D.F. Tel: 55 56222319. E-mail: marcegonf@yahoo.com.mx

${ }^{2}$ Doctora en Psicología. Facultad de Psicología, Universidad Nacional Autónoma de México. Profesora Titular Tiempo completo. Ave. Universidad 3004, Col. Copilco-Universidad, CP. 04510, Delegación Coyoacán, México, D.F. Tel: 55 56222319. E-mail: p.andradepalos@gmail.com
} 


\section{Introducción}

Los términos Bienestar Psicológico (BP) fueron empleados por primera vez en 1969 por Bradburn, cuando publicó los resultados de casi una década de investigaciones acerca de la felicidad o eudemonía. En su libro no sólo nombró el constructo sino que también sugirió un modelo de BP para explicar su estructura que, desde su punto de vista, estaba conformado por varias dimensiones de afecto positivo y negativo, por lo que propuso una escala de balance afectivo para medirlas (Bradburn, 1969).

El constructo de BP ha sido estudiado desde diversas perspectivas teóricas, que han identificado dos tendencias: la hedonista y la eudemonista (Keyes, Shmotkin, \& Ryff, 2002; Ryan \& Deci, 2001; Waterman, 1993). En cada una de éstas hay investigadores destacados que han aportado evidencia empírica para sustentar sus planteamientos, sin embargo, a pesar de la evidencia acumulada en el estudio de ambas concepciones no se ha logrado un consenso. Los ejes del enfoque hedonista o placentero han sido: la felicidad y el Bienestar Subjetivo (BS) constituido por el balance entre afecto positivo y negativo y la Satisfacción con la Vida (SV)(Diener, 1984; Diener, Emmons, Larsen, \& Griffin, 1985; Diener, Suh, Lucas, \& Smith, 1999; Kahneman, Diener, \& Schwarz, 1999). Por su parte los ejes del enfoque eudemonista se han centrado tanto en la búsqueda de la autorealización y la significación personal (Ryff,1989a, 1989b, 1995; Ryff \& Keyes, 1995; Ryff, Singer, \& Dienberg, 2004; Waterman, 1993; Waterman, Schwartz, \& Conti, 2008), como en el logro de la auto-actualización y de la autodeterminación (Deci \& Ryan, 1985, 2000, 2008; Ryan \& Deci, 2000, 2001; Ryan \& Huta, 2009).

La investigación empírica relacionada con estas dos perspectivas sugiere que estas concepciones del bienestar están relacionadas pero son diferentes, pues cada una mantiene sus propias características (Keyes et al., 2002; Waterman, 1993, 2008).

Algunos investigadores como Kashdan, Biswas-Diener y King (2008) han puntualizado los problemas y los costos de distinguir entre estas dos concepciones, ya que consideran que esta diferenciación tiene sus raíces en la filosofía, por lo que no sólo no ha podido ser definida, sino que su medición adolece de consistencia. Estos autores (Biswas-Diener \& King, 2009) han planteado que la distinción entre ambas perspectivas constituyen dos tradiciones de investigación del constructo pero no dos tipos de felicidad. En respuesta a este planteamiento, algunos académicos (Delle Fave \& Bassi, 2009; Keyes \& Annas, 2009; Ryan \& Huta, 2009; Waterman, 2008) han hecho énfasis en la importancia del estudio del pensamiento eudemonista. Uno de los argumentos más puntuales es el de Waterman (2008), quien ha estudiado desde hace varios años el Bienestar Eudemonista (BE), centrado en la calidad de vida de la persona - presencia y frecuencia de emociones positivas y negativas a lo largo del tiempo, así como un nivel general de satisfacción de vida (Waterman et al., 2013). Con respecto a la polémica acerca de la pertinencia de investigar las dos perspectivas, ha planteado que no hay suficiente evidencia empírica y que hasta ahora ninguna ha sido contundente para comprender la felicidad y el bienestar. Por ello es importante que se siga investigando en ambas perspectivas y en las diversas concepciones de bienestar, para distinguir los tres constructos de bienestar: el BS, el BP y el BE. La evidencia acumulada respecto de cada uno permitirá comprender qué es lo que hace que una vida se viva bien (Waterman, 2008).

Ryff (1989a, 1989b) fue una pionera en el estudio del funcionamiento positivo del ser humano y del bienestar psicológico desde la perspectiva eudemonista. Interesada en el funcionamiento positivo de las personas en la vejez, cuestionó las investigaciones y las mediciones acerca del BS, criticó la falta de sustento teórico de ese constructo y su énfasis en el afecto positivo y negativo. Además manifestó su desacuerdo con la traducción de eudemonía, ya que este término se había traducido como felicidad y, desde su punto de vista, estaba más relacionado con la búsqueda de autorrealización.

Sobre la base de las aportaciones de diversos teóricos de la psicología clínica, humanista, del desarrollo, del ciclo vital y de la salud mental, elaboró un modelo multidimensional de bienestar psicológico conformado por seis dimensiones: 1) 
Auto-aceptación: actitud positiva hacia el self, hacia buenas y malas cualidades y sentimientos positivos acerca de la vida pasada; 2) Relaciones positivas con otros: interacciones cálidas, satisfactorias, basadas en la confianza, alta empatía, intimidad, capacidad para dar y recibir; 3) Dominio del ambiente: dominio y competencia, control de arreglos complejos de actividades externas, empleo eficiente de oportunidades; 4) Autonomía: auto-determinación e independencia, capacidad de resistir presión social, control interno de la propia conducta, autoevaluación con criterios propios; 5) Propósito de vida: tener metas y un sentido de dirección, entender que la vida pasada y presente tiene significado, creer que la vida tiene sentido, propósitos y objetivos y 6) Crecimiento personal: sentimiento de continuo desarrollo, en crecimiento y expansión, apertura a nuevas experiencias, búsqueda de mejora con el tiempo.

Las escalas de BP que diseñó originalmente Ryff (1989b) estuvieron conformadas por 192 reactivos, 32 por cada dimensión; posteriormente presentó la versión de 9 ítems por dimensión y finalmente una versión corta de 18 reactivos, 3 reactivos por dimensión (Ryff \& Keyes, 1995), ésta última ha sido la más utilizada; sin embargo algunos investigadores han empleado un número diferente de reactivos en sus estudios por convenir así a sus propósitos de trabajo (Cheng \& Chang, 2005; Kafka \& Kozma, 2004; van Dierendock, 2005). Las escalas han sido utilizadas en diversas investigaciones tanto en Estados Unidos como en otros países principalmente con población adulta y en menor medida con jóvenes. En algunas se ha confirmado la multidimensionalidad del constructo (Akin, 2008; Díaz et al., 2006; Tomás, Meléndez, \& Navarro, 2008; Ryff \& Keyes, 1995; van Dierendonck, 2005), pero en otras no se han corroborado las seis dimensiones (Abbott et al., 2006; Burns \& Machin, 2009; Kafka \& Kozma, 2002; Springer \& Hauser, 2006; Springer, Hauser, \& Freese, 2006; Triadó, Villar, Solé, \& Celdrán, 2007).

En 2005, van Dierendonck realizó dos estudios con población alemana en 233 estudiantes de psicología ( $M=22$ años) y 420 profesionales ( $M=36$ años), uno de sus propósitos fue probar la validez factorial y de contenido de las escalas de BP de Ryff. Para hacerlo empleó las versiones de 3, 9 y 14 ítems por dimensión; sus resultados confirmaron únicamente la estructura de la versión corta de 18 ítems (3 por dimensión). Este investigador redujo los ítems por dimensión, de 14 a 6, 7 y ocho ítems. Su versión quedó conformada por un total de 39 ítems, los cuales miden las seis dimensiones propuestas originalmente por Ryff (1989a, 1989b), con una buena consistencia interna ( $\alpha=0.72$ a 0.81$)$; esta escala ha sido empleada en estudios realizados en España y México.

Las investigaciones desarrolladas con las escalas originales han correlacionado algunas de sus dimensiones con factores sociodemográficos como la edad y el sexo (Ryff, 1989a, 1989b; Ryff, 1995; Ryff \& Keyes, 1995; Ryff \& Heidrich, 1997; Ryff \& Singer, 1998). Algunos de estos estudios han mostrado que en la dimensión de autonomía, los participantes en edad adulta temprana y los adultos mayores obtuvieron puntajes más altos en comparación con los jóvenes. En el dominio del ambiente las puntaciones más altas fueron para los adultos tempranos y tardíos, y en propósito de vida y crecimiento personal los jóvenes y los adultos tempranos obtuvieron puntajes más altos. Respecto a las diferencias por sexo se encontró que las mujeres tuvieron puntajes más elevados que los varones en relaciones positivas con otros y en crecimiento personal (Abbott, et al, 2006; Clarke, Marshall, Ryff, \& Wheaton, 2001; Lindfors, Berntsson, \& Lundberg, 2006; Ryff, 1989b; Ryff, 1995; Ryff \& Singer, 2006; van Dierendock, 2005; Vleioras \& Bosma, 2005).

Ryff y Singer (2008) consideran que el contexto que rodea al individuo es fundamental para su auto-realización, desde su punto de vista el BP y el nivel educativo están vinculados positivamente, por lo que afirman que el propósito de vida y el crecimiento personal son los pilares de la eudemonía.

La evidencia empírica relacionada con el modelo multidimensional de BP con población de habla hispana se remonta al año 2000, con el trabajo de Casullo y Castro Solano (2000, 2001). Estos investigadores se basaron en las propuestas teóricas de Schmutte y Ryff (1997) para desarrollar una escala de bienestar BIEPS-J, que 
midiera el BP de adolescentes argentinos. Los participantes fueron 1270 estudiantes de secundaria de 13 a 18 años, de tres regiones geográficas de la República Argentina. Sus resultados mostraron que únicamente cuatro de las seis dimensiones propuestas por Ryff explicaron más del $50 \%$ de la varianza (control de situaciones, vínculos, proyectos y aceptación de sí mismo), si bien los índices de confiabilidad por dimensión fueron bajos $(\alpha=0.50$ a 0.56), la confiabilidad total fue aceptable $(\alpha=0.74)$. Las dimensiones de crecimiento personal y de autonomía fueron eliminadas. Esta escala mide cuatro dimensiones del BP, se ha aplicado en diversas investigaciones en Argentina (Casullo et al., 2002; Contini de González, Coronel de Pace, Levin, \& Esteves Suedan, 2003); además de confirmar su validez en países como Perú (Martínez \& Morote, 2002) y España (Cruz, Maganto, Montoya, \& González, 2002).

En España se han realizado también otros estudios empleando otras escalas, la primera versión en castellano de las escalas de Ryff fue desarrollada por Díaz et al. (2006), quienes tradujeron y adaptaron la versión propuesta por van Dierendonck (2005) conformada por seis dimensiones y 39 ítems. En esta investigación participaron 467 personas de 18 a 72 años $(M=33$ años; $D S=14)$. Las escalas de van Dierendonck mostraron una buena consistencia interna $(\alpha=0.68$ a 0.83) pero el nivel de ajuste de los análisis factoriales confirmatorios no fue satisfactorio. Al respecto desarrollaron una nueva versión que mantuvo las seis dimensiones y se redujo a 29 reactivos, con una buena consistencia interna $(\alpha=0.70$ a 0.84$)$. Otra investigación con población española es la de Triadó et al. (2007), quienes estudiaron la validez factorial y de contenido de la versión de 54 ítems (9 por dimensión) de las escalas de Ryff. Estos académicos trabajaron con 422 adultos mayores y en sus resultados reportaron que la consistencia interna de las escalas fue baja y que los análisis confirmatorios no comprobaron la estructura factorial del modelo de seis factores.

Por su parte, Tomás, Meléndez y Navarro (2008) analizaron la estructura factorial de la versión de 54 ítems de las escalas de Ryff; participaron 169 adultos, de entre 65 y 94 años de la comunidad Valenciana. Sus resultados mostraron que los valores de ajuste fueron similares a los reportados en la literatura que aporta evidencia para apoyar el modelo de seis factores con adultos mayores.

Ese mismo año, van Dierendonck, Díaz, Rodríguez-Carbajal, Blanco y Moreno-Jiménez (2008) realizaron un estudio, en el que aplicaron la adaptación de las escalas de Ryff, realizada por van Dierendonck (2005), su propósito fue obtener mayor información relacionada con la utilidad de estas escalas en una cultura diferente a la anglosajona. Participaron 919 personas, de las cuales, 592 eran españoles y 327 originarios de Columbia y el rango de edad fue de 16 a 74 años $(M=30$ años; $D S=14)$. Sus resultados confirmaron la utilidad del modelo para una cultura no anglosajona, y sugirieron también mantener las seis escalas intactas al emplearlas en otras culturas.

En México, Balcázar, Bonilla, Gurrola, Loera y Trejo (2009) adaptaron la escala de van Dierendonck (2005), traducida al castellano por Díaz et al. (2006). Participaron 208 estudiantes mexicanos, de 14 a 18 años (50\% hombres y 50\% mujeres) de una preparatoria del Estado de México. El instrumento empleado midió seis dimensiones y estuvo conformado por 39 reactivos. Los resultados del análisis factorial no confirmaron el modelo de seis factores, sólo se agruparon cuatro factores: auto-aceptación con 15 ítems $(\alpha=0.91)$; relaciones interpersonales con ocho ítems $(\alpha=0.75)$; autonomía con seis ítems $(\alpha=0.72)$ y satisfacción con la vida, que agrupó cinco ítems $(\alpha=0.63)$; estos factores explicaron el $41.79 \%$ de varianza integraron 34 ítems de la escala original, las dimensiones de autonomía y dominio del ambiente se eliminaron. Cabe mencionar que el cuarto factor que estos autores denominaron satisfacción con la vida, no corresponde a ninguna de las dimensiones propuestas por Ryff (1989, 1989b), en realidad estos reactivos corresponden a la dimensión de crecimiento personal.

Otro trabajo con población mexicana es el de Medina-Calvillo, Gutiérrez-Hernández y PadrósBlázquez (2013), quienes también estudiaron las propiedades psicométricas de la versión de van Dierendonck (2005), traducidas por Díaz et al. 
(2006). Esta versión, como se mencionó anteriormente cuenta con 39 reactivos y mide las seis dimensiones propuestas por Ryff. Estos investigadores trabajaron con dos muestras: en la muestra A: participaron 477 alumnos universitarios ( $M=19$ años) y en la muestra B: 256 personas de diversas edades $(M=29.46$ años; $D S=11.36$ ). Sus resultados mostraron que la escala no se ajustó al modelo de 6 factores, reportaron una alta consistencia interna para el total de la escala en la muestra A $(\alpha=0.91)$ y valores alpha moderados para cada una de las seis dimensiones $(\alpha=0.61$ a 0.79$)$; en la muestra B la consistencia interna para el total de la escala fue alta $(\alpha=0.90)$, pero los valores alpha de cada una de las seis dimensiones fueron bajos $(\alpha=0.39$ a 0.72).

La controversia en los resultados acerca de la multidimensionalidad del constructo y la utilidad del modelo en una cultura no anglosajona; aunado al hecho de que, en muchos de estos estudios se ha trabajado con población adulta (mayores de 21 años) y en menor medida con estudiantes menores de 18 años (Balcazar et al., 2009; Casullo \& Castro-Solano, 2000, 2001; Medina-Calvillo et al., 2013; Ryff, 1989b; van Dierendonck, 2005), hace necesario continuar investigándolo, sobre todo si se estudia en una cultura diferente a la norteamericana. Este planteamiento concuerda con el punto de vista de Ryff (1989b), quien señaló la importancia de considerar las diferencias culturales al emplear sus escalas, ya que sus resultados correspondían a los valores de una clase social media alta de los Estados Unidos.

Los propósitos de este trabajo fueron diseñar y validar una escala para evaluar la percepción de $\mathrm{BP}$ en adolescentes mexicanos. La base teórica en la que se fundamenta este trabajo fue el modelo multidimensional de BP de Ryff (1989a, 1989b).

La investigación fue de tipo ex post facto y se desarrolló en dos fases, la primera se centró en el diseño y la validación de la escala de BP y la segunda permitió confirmar sus propiedades psicométricas.

\section{Fase I}

Esta fase fue de tipo exploratorio y se dividió en dos estudios: el primero tuvo como propósito identificar el significado que los adolescentes le asignaron a los términos $\mathrm{BP}$ y el segundo se centró en el diseño y la validación de la escala.

\section{Estudio 1. Significado de BP}

Sus objetivos fueron: a) identificar el significado que tiene para algunos adolescentes los términos bienestar y BP, y b) identificar los aspectos/dimensiones individuales y contextuales que asocian los adolescentes como necesarios para favorecer el $\mathrm{BP}$, así como aquéllos que lo obstaculizan.

\section{Método}

\section{Participantes}

Los participantes fueron 30 estudiantes (19 hombres y 11 mujeres), de 15 a 20 años de edad, inscritos en escuelas públicas de Educación Media Superior (EMS), de la ciudad de México. Se organizaron tres grupos de diez alumnos cada uno, el grupo 1 se conformó por 5 hombres y 5 mujeres; el grupo 2, por cinco hombres y cinco mujeres, y el grupo 3, por 9 hombres y una mujer.

\section{Técnica para la obtención de la información}

Para identificar el significado que le dieron los adolescentes a los términos relacionados con el BP, se empleó la técnica de grupo focal (Krueger \& Casey, 2000), que permitió la discusión y el intercambio de ideas entre los participantes. La organización y conducción de las sesiones se realizó con base en un guión previamente elaborado, en donde se incluyeron preguntas estímulo basadas en los planteamientos del modelo multidimensional de Ryff (1989a, 1989b).

\section{Procedimiento}

Para solicitar la autorización, se informó a las autoridades y a los profesores de las escuelas el propósito del estudio. La participación de los jóvenes fue voluntaria y anónima, se les explicó: el propósito del estudio, la forma de trabajo en el grupo, la duración de la sesión (una hora), además de indicarles que se tomarían notas y se grabaría en audio. La actividad con los grupos se ajustó a lo estipulado por Krueger y Casey (2000) para trabajar con adolescentes (un mínimo de 3 grupos, con una duración máxima de una hora por grupo). 


\section{Análisis de la información}

Se resumieron e integraron las descripciones de los jóvenes para cada pregunta estímulo (Kvale, 2007). La información se transcribió textualmente para analizar las respuestas, posteriormente se agruparon las descripciones cuyo contenido era similar.

\section{Resultados}

Las descripciones que proporcionaron los jóvenes se organizaron en cinco rubros: sentirse bien, bienestar, BP, aspectos necesarios para tener BP y aspectos que obstaculizan el BP. Se identificaron dos elementos importantes: los aspectos personales (emociones, conductas, sentimientos) y los contextuales (familia, paresamigos y/o novio, escuela y sociedad), los primeros se presentan en la Figura 1 y los contextuales en la Figura 2.

\begin{tabular}{c}
$\begin{array}{c}\text { Figura 1. Términos empleados por los jóvenes } \\
\text { para describir el significado de BP }\end{array}$ \\
\hline Aspectos personales \\
\hline
\end{tabular}

Es más mental que físico, la parte mental y física están unidas, si una falla la otra también puede fallar.

Controlar tus emociones y sentimientos.

Autoconocimiento, auto-concepto (aceptar lo que somos, conocer limitaciones, virtudes y defectos), conocerte bien.

Aceptarte.

Estar bien contigo mismo. Saber que estás haciendo las cosas bien.

Estar bien con emociones y acciones (congruencia).

Tener orden en lo que tenemos que hacer. Saber por qué lo haces.

Tiene que ver con la autoestima (que tan contento/a gusto/bien estás contigo mismo). Es la capacidad para querernos y aceptarnos, conocerse a sí mismo (virtudes, defectos) no tener problemas con lo que no puedes hacer.)

Es lo interno y lo social.

Tener algún tipo de motivación.

Tener estabilidad emocional (sentimientos y pensamientos).

Tener equilibrio (coordinar) entre lo que piensas y haces para elegir.

Tener confianza en ti mismo para planear tus metas y alcanzarlas, mediante la determinación que te impongas en ti mismo.

Tener seguridad en ti mismo, en lo que quieres a futuro. Tener criterio propio para tomar decisiones.

No estar solos, no estar estresados.

No tener desequilibrio emocional (no tener remordimientos).

\begin{tabular}{|c|c|c|c|}
\hline \multicolumn{4}{|c|}{$\begin{array}{l}\text { Figura 2. Términos empleados por los jóvenes } \\
\text { para describir el significado de BP }\end{array}$} \\
\hline \multicolumn{4}{|c|}{ Aspectos contextuales } \\
\hline Familiares & $\begin{array}{c}\text { Pares, } \\
\text { amigos/as, } \\
\text { novios/as }\end{array}$ & Escolares & Sociales \\
\hline $\begin{array}{c}\text { El trato de } \\
\text { la familia. } \\
\text { La unión } \\
\text { familiar, } \\
\text { el apoyo, } \\
\text { consejos } \\
\text { para estar } \\
\text { mejor. } \\
\text { La } \\
\text { seguridad } \\
\text { desde la } \\
\text { casa. } \\
\text { Tomar lo } \\
\text { bueno de } \\
\text { la familia. }\end{array}$ & $\begin{array}{c}\text { Estar con } \\
\text { alguien de } \\
\text { confianza, } \\
\text { con tus } \\
\text { amigos. } \\
\text { Sentir que } \\
\text { todo fluye } \\
\text { con las } \\
\text { personas } \\
\text { que } \\
\text { convivo. } \\
\text { No tener } \\
\text { problemas } \\
\text { con la } \\
\text { pareja: } \\
\text { novio/a. } \\
\text { Ayudar a } \\
\text { otros. }\end{array}$ & $\begin{array}{c}\text { Es } \\
\text { importante } \\
\text { en nuestras } \\
\text { vidas, tan } \\
\text { importante } \\
\text { como la } \\
\text { familia. } \\
\text { Da } \\
\text { seguridad, } \\
\text { confianza } \\
\text { y permite } \\
\text { tener una } \\
\text { mejor } \\
\text { experiencia } \\
\text { de vida. }\end{array}$ & $\begin{array}{c}\text { La } \\
\text { sociedad, } \\
\text { lo que } \\
\text { pasa en } \\
\text { el } \\
\text { entorno } \\
\text { te afecta. }\end{array}$ \\
\hline
\end{tabular}

El resumen de las palabras que los adolescentes asociaron con BP se presenta en la Figura 3. Algunas de las palabras que los jóvenes emplearon con más frecuencia fueron: aceptarse, auto-conocimiento, estar bien consigo mismo, logros, disfrutar lo que tengo, autoestima y seguridad en ti mismo.

Las descripciones de los adolescentes se compararon con las definiciones conceptuales y operacionales de las seis dimensiones propuestas por Ryff (1989b). Las descripciones de los jóvenes se clasificaron en cinco de las seis dimensiones del modelo multidimensional de BP, con excepción de la dimensión de dominio del ambiente, que no fue identificada claramente por los participantes. Se revisaron nuevamente las descripciones y se identificó que varias de las aportaciones de los adolescentes hicieron alusión a la importancia del control personal para tener BP, por lo que se decidió cambiar dominio del ambiente por control personal. En la primera columna de la Figura 4, se presentan las definiciones conceptuales de Ryff, en la segunda las descripciones proporcionadas por los 
Figura 3. Palabras asociadas por los jóvenes con BP

No estar loco

Estar bien consigo mismo

Aspectos emocionales

Logros

Autonomía

Disfrutar lo que tengo

Analizar lo que te dicen otros y decidir

Autoestima

Aceptarse

Ver otras ideas (cambiar)

Seguridad en ti mismo

Influencia de todos (familia, amigos, compañeros)

El tipo de pensamientos de la persona (positivos)

Personalidad

Autoconocimiento (lo bueno, lo malo y los miedos)

Establecimiento y logro de metas continuo

Desarrollo personal

Felicidad

Sin desgaste mental por presiones

Estabilidad

Sin estrés (no demasiado, un poco es bueno)

Tiempo y gusto para hacer lo que quieres

Cultura

Inteligencia para superarte a ti mismo

Sabiduría

El bienestar que te dan los conocimientos

Superarte a ti mismo

Seguridad en lo que hacemos

Inteligencia, seguridad para solucionar problemas

adolescentes y en la tercera las definiciones adaptadas para adolescentes, en la última fila se mantuvo la definición operación de Ryff respecto a domino del ambiente, pero se hizo el cambio a control personal en las descripciones de los jóvenes y en la definición adaptada a población mexicana.

Con los resultados obtenidos en este estudio se redactaron los reactivos para cada una de las seis dimensiones, la escala quedó conformada por 134 ítems.
Estudio 2. Diseño y validación de la escala de Bienestar Psicológico para Adolescentes (BPA).

El objetivo fue analizar las características psicométricas de la escala de BP para Adolescentes (BP-A).

\section{Método}

\section{Participantes}

La muestra fue no probabilística y estuvo conformada por 665 estudiantes de escuelas públicas de EMS de la ciudad de México, el rango de edad fue de 14 a 20 años $(M=16.7 ; D E=1.36)$, el $50 \%$ fueron mujeres y el otro $50 \%$ varones. El $64 \%$ vivía con ambos padres, $28.6 \%$ únicamente con la madre, el 2.3 sólo con su padre y el resto $4.1 \%$ con algún otro familiar.

\section{Instrumento}

Escala de Bienestar Psicológico para Adolescentes (BP-A), diseñada en el estudio anterior. La escala es auto-aplicable y mide la percepción de BP, está conformada por 134 reactivos, distribuidos en seis dimensiones: Autoaceptación con 27 reactivos, proyecto de vida con 24 reactivos, crecimiento personal con 19 reactivos, relaciones positivas con otros con 22 reactivos, autonomía con 19 reactivos y control personal con 23 reactivos. Se utilizó el método de escalamiento de tipo Likert, con cuatro opciones de respuesta (totalmente de acuerdo, de acuerdo, en desacuerdo y totalmente en desacuerdo). El tiempo de aplicación fue de aproximadamente 30 minutos.

\section{Procedimiento}

La escala BP-A se aplicó directamente en los salones de clase, previa autorización de los directivos y los profesores. La participación de los jóvenes fue voluntaria y anónima, se les proporcionó información respecto al propósito del estudio, la manera de contestarlo y el tiempo aproximado para hacerlo, también se les aclararon sus dudas. 
Figura 4. Definiciones de las dimensiones de BP de Ryff (1989a,b), significado atribuido por los estudiantes y definiciones adaptadas para adolescentes mexicanos

\begin{tabular}{|c|c|c|}
\hline $\begin{array}{c}\text { Definiciones conceptuales } \\
\text { Ryff }(1989 a, b)\end{array}$ & $\begin{array}{l}\text { Significado atribuido por los } \\
\text { estudiantes para describir el BP }\end{array}$ & $\begin{array}{c}\text { Definiciones de cada dimensión } \\
\text { de BP adaptadas }\end{array}$ \\
\hline $\begin{array}{l}\text { Auto-aceptación } \\
\text { Característica central de salud mental, de la } \\
\text { auto-actualización, del funcionamiento } \\
\text { óptimo y la madurez. Las teorías del ciclo } \\
\text { vital hacen énfasis en la aceptación del self } \\
\text { y de la vida pasada. }\end{array}$ & $\begin{array}{c}\text { Auto aceptación } \\
\text { Autoconocimiento } \\
\text { Autoestima } \\
\text { Confianza/seguridad en sí mismos. } \\
\text { Controlar tus emociones y } \\
\text { sentimientos }\end{array}$ & $\begin{array}{l}\text { Auto-aceptación } \\
\text { Aceptación o rechazo de sus } \\
\text { capacidades y limitaciones } \\
\text { personales. }\end{array}$ \\
\hline $\begin{array}{c}\text { Relaciones positivas con otros } \\
\text { Establecer vínculos interpersonales cálidos } \\
\text { y confiables. La habilidad para amar es } \\
\text { componente central de la salud mental. Los } \\
\text { que se auto-actualizan se describen con } \\
\text { fuertes sentimientos de empatía y afecto por } \\
\text { todos. Las teorías del desarrollo hacen } \\
\text { énfasis en el logro de la intimidad y la } \\
\text { generatividad. }\end{array}$ & $\begin{array}{l}\text { Interacciones positivas con } \\
\text { familia, amigos, compañeros, } \\
\text { novio/a y gente. } \\
\text { Ayudar a otros } \\
\text { Compartir }\end{array}$ & $\begin{array}{l}\text { Interacciones positivas con } \\
\text { otros } \\
\text { Percepción del adolescente } \\
\text { respecto a sus } \\
\text { habilidades/dificultades para } \\
\text { establecer relaciones respetuosas } \\
\text { y cordiales con los otros. }\end{array}$ \\
\hline $\begin{array}{c}\text { Propósito de vida } \\
\text { La salud mental incluye creencias de que } \\
\text { hay un propósito y un significado para la } \\
\text { vida. La madurez hace énfasis en una clara } \\
\text { comprensión de un propósito de vida, un } \\
\text { sentido de direccionalidad e } \\
\text { intencionalidad, Las teorías del desarrollo } \\
\text { refieren cambios en los propósitos o metas } \\
\text { en la vida. }\end{array}$ & $\begin{array}{l}\text { Identificar metas personales } \\
\text { Tener confianza para planear tus } \\
\text { metas y alcanzarlas. } \\
\text { Seguridad en lo que quieres a } \\
\text { futuro. } \\
\text { Tener metas claras y realistas, } \\
\text { enfocarse en ellas. } \\
\text { Tener orden en lo que tenemos que } \\
\text { hacer, saber por qué lo haces. }\end{array}$ & $\begin{array}{c}\text { Proyecto de vida } \\
\text { Percepción del adolescente sobre } \\
\text { la relevancia de establecer } \\
\text { metas/objetivos personales a } \\
\text { futuro y habilidad para relacionar } \\
\text { sus esfuerzos actuales con el } \\
\text { logro de metas en los próximos } \\
\text { años. }\end{array}$ \\
\hline $\begin{array}{c}\text { Autonomía } \\
\text { Comprende las cualidades de auto- } \\
\text { determinación, independencia y regulación } \\
\text { de la conducta, con un locus interno de } \\
\text { evaluación que permite no depender de } \\
\text { otros para obtener aprobación. }\end{array}$ & $\begin{array}{c}\text { Autonomía, libertad, no depender } \\
\text { de otros para tomar decisiones } \\
\text { propias. } \\
\text { Seguridad en ti mismo, en lo que } \\
\text { quieres a futuro. } \\
\text { Tomar decisiones, aceptar las } \\
\text { consecuencias (sentirse libre, te } \\
\text { hace crecer, ser autónomo. } \\
\text { Tener criterio propio para tomar } \\
\text { decisiones. }\end{array}$ & $\begin{array}{c}\text { Autonomía } \\
\text { Percepción del adolescente para } \\
\text { tomar decisiones propias, } \\
\text { basadas en la reflexión personal } \\
\text { sobre las consecuencias positivas } \\
\text { y negativas de la elección, } \\
\text { independientemente de la presión } \\
\text { social de sus pares y sus padres. }\end{array}$ \\
\hline $\begin{array}{l}\text { Crecimiento Personal } \\
\text { Un funcionamiento óptimo requiere } \\
\text { continuar desarrollando su propio potencial } \\
\text { para crecer y expandirse como persona. Las } \\
\text { teorías del ciclo de vida hacen énfasis en el } \\
\text { crecimiento continuo y en la confrontación } \\
\text { de nuevos desafíos o tareas en todas las } \\
\text { etapas de vida. }\end{array}$ & $\begin{array}{c}\text { Desarrollo Personal Continuo } \\
\text { Hacer las cosas lo mejor que } \\
\text { puedas. } \\
\text { Identificar defectos y mejorarlos, } \\
\text { ser la mejor persona posible. } \\
\text { Superación académica. } \\
\text { Corregir errores. } \\
\text { Superar momentos difíciles. } \\
\text { Actitud positiva. }\end{array}$ & $\begin{array}{c}\text { Crecimiento Personal } \\
\text { Disposición, apertura e interés } \\
\text { del adolescente para informarse } \\
\text { continuamente y desarrollar su } \\
\text { potencial y sus capacidades } \\
\text { personales. }\end{array}$ \\
\hline $\begin{array}{c}\text { Dominio del Ambiente } \\
\text { Habilidad personal para elegir y crear } \\
\text { ambientes deseables para mantener las } \\
\text { condiciones psíquicas, es una característica } \\
\text { de la salud mental. }\end{array}$ & $\begin{array}{c}\text { Control Personal } \\
\text { Controlar tus emociones y } \\
\text { sentimientos } \\
\text { Estar bien con emociones y } \\
\text { pensamientos } \\
\text { Tener estabilidad emocional } \\
\text { Seguridad en lo que hacemos. }\end{array}$ & $\begin{array}{l}\text { Control Personal } \\
\text { Percepción del adolescente } \\
\text { respecto a su capacidad para } \\
\text { controlar sus impulsos, carácter y } \\
\text { comportamiento, aun cuando } \\
\text { esté alterado emocionalmente. }\end{array}$ \\
\hline
\end{tabular}




\section{Resultados}

Para realizar el análisis psicométrico de la escala se hicieron análisis factoriales con rotación varimax para cada dimensión. Los criterios para considerar un factor fueron: que tuviera tres ítems como mínimo, que presentaran una carga factorial superior a 0.40 y que tuvieran claridad conceptual.

El resultado de estos análisis permitió conformar una escala de 91 reactivos, distribuidos de la siguiente manera: 18 para auto-aceptación, 14 para proyecto de vida, 15 para crecimiento personal, 14 para relaciones positivas con otros, 18 para control personal y 12 para autonomía.

\section{Fase II}

El objetivo fue confirmar las propiedades psicométricas de la escala de BP para adolescentes mexicanos.

\section{Método}

\section{Participantes}

La selección de los participantes fue no probabilística, la muestra inicial fue de 1064 adolescentes de 15 a 20 años, estudiantes de escuelas públicas de EMS. Los cuestionarios incompletos fueron eliminados, por lo que la muestra final quedó conformada por 940 adolescentes: 427 (45.5\%) fueron varones y 513 (54.6\%) mujeres, el rango de edad fue de 15 a 20 años $(M=16.1 ; D E=0.94)$. El $72.3 \%$ vivía con sus padres, $21.3 \%$ con la madre, $2.7 \%$ con el padre y el resto $3.5 \%$ con algún otro familiar.

\section{Instrumentos}

Escala de Bienestar Psicológico para adolescentes (BP-A) conformada por 91reactivos, tipo Likert, con cuatro opciones de respuesta, construida y validada en la fase anterior. En esta versión los reactivos se presentaron de forma aleatoria.

\section{Procedimiento}

La escala BP-A se aplicó directamente en los salones de clases, previa autorización de los directivos y profesores de las escuelas. La participación de los estudiantes fue voluntaria y anónima, se les informó el propósito del estudio y se les aclararon sus dudas.

\section{Resultados}

Para confirmar la validez psicométrica de esta escala, primero se realizó una correlación ítemtotal con los reactivos de cada dimensión. En este análisis se tomó en cuenta el valor de alpha, si el elemento era eliminado. El criterio para seleccionar los reactivos por dimensión fue que obtuvieran una correlación ítem-total superior a 0.50 . Una vez realizado este análisis quedaron 61 reactivos. La dimensión de autonomía de eliminó porque sus reactivos no cumplieron con este criterio.

Para corroborar la dimensionalidad de la escala se realizó un análisis factorial con rotación varimax con los reactivos que cumplieron con el criterio antes descrito. Se eligieron los factores que presentaron un valor eigen superior a 1 , que incluyeran al menos tres ítems con una carga factorial superior a $0.50 \mathrm{y}$ que tuvieran claridad conceptual (Kaiser-Meyer-Olkin $=0.911$, Prueba Barlett Chi-cuadrado aproximado $=12$ 032.95, $g l=$ 496). El resultado de este análisis permitió identificar siete factores, que explicaron el $60.51 \%$ de la varianza total. En la Tabla 1 se presentan los factores, el número de reactivos y su consistencia interna.

Los resultados de las correlaciones entre los siete factores de la escala de BP se presentan en la Tabla 2, la fuerza de la relación entre estos factores fue moderada, lo que sugiere que están relacionados pero cada uno mide constructos diferentes.

\section{Discusión}

El propósito central de este estudio se cumplió, se diseñó y validó psicométricamente la escala de BP para adolescentes mexicanos, basada en el modelo multidimensional de BP de Ryff. La escala BP-A se sustentó teórica y empíricamente, ya que para su diseño se consideraron tanto las descripciones de los adolescentes para explicar el significado del BP, como los supuestos teóricos y las definiciones conceptuales de Ryff (1989b). 
Tabla 1. Propiedades psicométricas de la Escala de Bienestar Psicológico para Adolescentes (BP-A)

\begin{tabular}{|c|c|c|}
\hline 7 Factores & 29 Reactivos & $\begin{array}{c}\text { Peso } \\
\text { Factoria }\end{array}$ \\
\hline F1. CRECIMIENTO & 1. Estoy interesado(a) en adquirir habilidades nuevas. & .735 \\
\hline $\begin{array}{l}\text { PERSONAL } \\
\text { Reactivos } 6\end{array}$ & $\begin{array}{l}\text { 2. Estoy abierto(a) a nuevas experiencias que contribuyan a mi } \\
\text { formación personal. }\end{array}$ & .697 \\
\hline$\alpha=0.79$ & 3. Me intereso en perfeccionar mis habilidades. & 655 \\
\hline Varianza explicada: & 4. Mi actitud es de apertura al conocimiento y la innovación. & .591 \\
\hline $26.47 \%$ & 5. Estudio para saber más y enfrentar los retos de la vida. & .564 \\
\hline$p=.000$ & 6. Si me esfuerzo logro lo que quiero. & .518 \\
\hline F2. RELACIONES & 1. Me relaciono fácilmente con personas de mi edad. & .799 \\
\hline POSITIVAS CON & 2. Se me hace difícil hacer nuevos amigos (as). & $.782 *$ \\
\hline OTROS & 3. Me cuesta trabajo relacionarme con las personas. & $.753^{*}$ \\
\hline $\begin{array}{l}\text { Reactivos } 5 \\
\alpha=0.81\end{array}$ & 4. Tengo buenas relaciones con mis compañeros (as). & .662 \\
\hline $\begin{array}{l}\text { Varianza explicada: } \\
10.15 \% \\
p=.000\end{array}$ & 5. Me llevo bien con mis compañeros (as). & 647 \\
\hline F3. PROYECTO DE & 1. Tengo metas claras sobre lo que quiero hacer en mi vida & .774 \\
\hline $\begin{array}{l}\text { VIDA } \\
\text { Reactivos } 4\end{array}$ & $\begin{array}{l}\text { 2. Tengo un plan sobre lo que quiero hacer con mi vida los próximos } \\
\text { años. }\end{array}$ & .761 \\
\hline $\begin{array}{l}\alpha=0.80 \\
\text { Varianza explicada: }\end{array}$ & 3. Tengo un plan de vida que le da dirección y guía a mis acciones. & .730 \\
\hline $\begin{array}{l}6.59 \% \\
p=.000\end{array}$ & 4. Me he propuesto lograr varias metas. & .540 \\
\hline F4. AUTO- & 1. Me quiero con todos mis defectos. & .784 \\
\hline $\begin{array}{l}\text { ACEPTACIÓN } \\
\text { Reactivos } 4\end{array}$ & 2. Acepto mis defectos. & .728 \\
\hline $\begin{array}{l}\alpha=.74 \\
\text { Varianza explicada: }\end{array}$ & 3. Quisiera tener un cuerpo diferente. & $.678^{*}$ \\
\hline $\begin{array}{l}5.62 \% \\
p=.000\end{array}$ & 4. Odio mis defectos. & $.567 *$ \\
\hline F5. PLANES A & 1. Hacer planes para el futuro es perder el tiempo. & $.711^{*}$ \\
\hline $\begin{array}{l}\text { FUTURO } \\
\text { Reactivos } 4\end{array}$ & 2. Hacer un proyecto de vida es una pérdida de tiempo. & $.732 *$ \\
\hline $\begin{array}{l}\alpha=.77 \\
\text { Varianza explicada: }\end{array}$ & 3. Para lograr lo que quiero es importante hacer planes. & .613 \\
\hline $\begin{array}{l}4.46 \% \\
p=.000\end{array}$ & 4. Si lucho por alcanzar mis metas las alcanzaré. & .503 \\
\hline F6. RECHAZO & 1. Odio mi carácter. & $.815^{*}$ \\
\hline $\begin{array}{l}\text { PERSONAL } \\
\text { Reactivos } 3 \\
\alpha=0.77\end{array}$ & 2. Odio mi manera de ser. & $.736 *$ \\
\hline $\begin{array}{l}\text { Varianza explicada: } \\
3.85 \% \\
p=.000\end{array}$ & 3. Quisiera tener otro carácter. & $.721 *$ \\
\hline $\begin{array}{l}\text { F7. CONTROL } \\
\text { PERSONAL }\end{array}$ & 1. Controlo mi comportamiento aunque esté alterado (a). & .826 \\
\hline $\begin{array}{l}\text { Reactivos } 3 \\
\alpha=0.83\end{array}$ & 2. Puedo controlar mis impulsos. & .823 \\
\hline $\begin{array}{l}\text { Varianza explicada: } \\
3.40 \% \\
p=.000\end{array}$ & 3. Domino fácilmente mi carácter. & .800 \\
\hline
\end{tabular}

El resultado de este proceso fue la adaptación de las definiciones conceptuales de cada dimensión para los adolescentes que participaron en el estudio. El procedimiento para la validación psicométrica permitió depurar el número de ítems y las dimensiones que finalmente conformaron la versión final de la escala. Los índices de consistencia interna para la escala total $(\alpha=0.76)$ y para cada una de las siete dimensiones fueron adecuados (de 0.74 a 0.83 ), por lo se puede afirmar que se cuenta con un instrumento válido y confiable para medir el BP en adolescentes mexicanos, desde una perspectiva eudemonista. Por otro lado, las correlaciones entre las seis dimensiones de la escala de BP-A fueron positivas, con excepción de la correspondiente 
Tabla 2. Índices de correlación entre las 7 dimensiones de la escala de BP-A

\begin{tabular}{|c|c|c|c|c|c|c|c|}
\hline & CreP & RPO & PV & $\mathrm{Aa}$ & $\mathrm{PF}$ & RchP & ConP \\
\hline CreP & 1 & & & & & & \\
\hline RPO & $.301^{* *}$ & 1 & & & & & \\
\hline PV & $.546^{* * *}$ & $295^{* *}$ & 1 & & & & \\
\hline $\mathrm{Aa}$ & $.302^{* * *}$ & $.338^{* *}$ & $.250^{* *}$ & 1 & & & \\
\hline PF & $.558^{* * *}$ & $274^{* * *}$ & $.585^{* * *}$ & $.239^{* *}$ & 1 & & \\
\hline RchP & $-.264^{*}$ & $-.310^{* *}$ & $-.241^{*}$ & $-.575^{* *}$ & $-.240^{* * *}$ & 1 & \\
\hline ConP & $.302^{* *}$ & $217^{* *}$ & $.268^{* *}$ & $.322^{* * *}$ & $.184^{* *}$ & $.395^{* * *}$ & 1 \\
\hline
\end{tabular}

Nota: $N=929 ; \quad \mathrm{CreP}=$ Crecimiento Personal; $\mathrm{RPO}=$ Relaciones Positivas con Otros; PV= Proyecto de Vida; $\mathrm{Aa}=$ Auto-aceptación; $\mathrm{PF}=$ Planes a Futuro; RchP= Rechazo Personal; ConP= Control Personal.

$* * p=>.01$

a rechazo personal que presentó una correlación negativa. La fuerza de la relación fue moderada, se observó una diferencia entre las siete dimensiones, lo que sugiere que son distintos aspectos de un mismo constructo.

Con relación al modelo multidimensional de $\mathrm{BP}$, en esta investigación no se confirmaron las seis dimensiones propuestas por Ryff. La primera dimensión del modelo multidimensional que se eliminó fue dominio del ambiente, ya que ningún joven mencionó descripciones relacionadas con esta dimensión (estudio1, fase I). Después de revisar las aportaciones de los mismos adolescentes, se decidió incluir el constructo de control personal. Este constructo ha sido investigado por diversos autores con nombres diferentes (locus de control, maestría, eficacia personal, auto-eficacia, auto-dirección y autonomía personal, entre otros), al respecto señalan que si bien estos constructos son diferentes, se traslapan en gran medida (Lewis et al., 1999).

La segunda dimensión que se eliminó por no cubrir los criterios de validación estipulados en este estudio fue autonomía (fase II), este resultado coincide con lo reportado por Casullo y Castro (Casullo, 2002; Casullo \& Castro, 2000) con adolescentes argentinos, en donde también se quitó esta dimensión porque resultó confusa para los jóvenes con quienes trabajaron.

Como se mencionó anteriormente, en esta investigación sólo se identificaron cuatro dimensiones de la propuesta original de Ryff: crecimiento personal, relaciones positivas con otros, auto-aceptación y propósito de vida. Más aún, en estas dos últimas dimensiones los ítems diseñados para cada una de ellas no se agruparon en un solo factor, sino en dos factores: autoaceptación se dividió en un factor que describe los aspectos positivos y otro factor que se denominó rechazo personal que se enfoca en los aspectos negativos. En cuanto a propósito de vida, apareció una situación semejante, se identificaron dos factores uno relacionado propiamente con el propósito de vida, con dos ítems positivos y dos negativos y el otro fue planeación a futuro, con dos ítems positivos y dos negativos. De esta manera la escala quedó conformada por 7 dimensiones y 29 reactivos.

Estos resultados hacen evidentes las diferencias tanto en el número como en las dimensiones que conforman la escala de BP-A y las diseñadas y/o adaptadas por algunos investigadores en otros países (Casullo, 2002; Casullo \& Castro, 2000; Díaz et al., 2006; Ryff, 1989a, Ryff, 1989b; Ryff \& Keyes, 1995).

La escala BIEPS-J para adolescentes argentinos (Casullo, 2002; Casullo \& Castro, 2000, Casullo et al., 2002) es diferente de la mexicana, no sólo por el proceso de diseño y validación, sino también por los resultados encontrados. Si bien en ambos casos no se confirma el modelo de seis factores y se elimina la dimensión de autonomía; en la escala BP-A desarrollada en este estudio se mantiene la dimensión de crecimiento personal, que no aparece en la escala argentina y que de acuerdo con Ryff y Singer (2008) es uno de los pilares de la autorrealización. Otras diferencias son el número de dimensiones: cuatro (control, vínculos, proyectos y aceptación) versus siete (crecimiento personal, relaciones positivas con otros, propósito de vida, auto-aceptación, planes a futuro, rechazo personal y control personal), el número de reactivos: 13 versus 29, los índices de confiabilidad por dimensión: $(\alpha=0.50$ a 0.56$)$ versus $(\alpha=0.74$ a 0.83$)$, y la confiabilidad total de cada escala: $\alpha=0.74$ versus 0.76 .

Los resultados de esta investigación coinciden con los de Balcázar et al. (2009) y con los de Medina-Calvillo et al. (2013), pues en ninguno de estos estudios con población mexicana, sus resultados se ajustaron al modelo de seis factores. En sus conclusiones Medina-Calvillo et al. (2013) 
plantearon la necesidad de realizar propuestas alternativas que se ajustaran mejor a los adolescentes mexicanos.

Un resultado que llama la atención es que tanto en Argentina como en México se haya eliminado la dimensión de autonomía. Este constructo es clave en el estudio de la adolescencia, sin embargo en las culturas latinoamericanas, en donde los adolescentes viven y dependen de sus padres, esta dimensión resulta ser un constructo confuso para los jóvenes (Casullo et al, 2002), por lo que es necesario realizar más investigación al respecto. Los resultados de los estudios realizados en Argentina y México no coinciden totalmente con los obtenidos por van Dierendonck et al. (2008), ya que si bien el modelo parece ser útil en una cultura diferente a la anglosajona, no se mantuvieron las seis escalas propuestas por Ryff (Balcázar et al., 2009; Casullo, 2002; MedinaCalvillo et al., 2013).

Con relación al constructo de crecimiento personal, llama la atención que se haya eliminado en las investigaciones con estudiantes argentinos y que en este estudio haya sido una de las dimensiones que conformaron el BP. En futuras investigaciones será necesario continuar estudiando si esta dimensión se mantiene en poblaciones de adolescentes activos laboralmente y que no están estudiando.

Ryff (1989b) planteó que las diferencias culturales constituyen un elemento central en el estudio del BP, sus escalas fueron desarrolladas para una clase social media alta de los Estados Unidos, por lo que advirtió a los investigadores acerca de la necesidad de considerar las diferencias culturales, sociales, éticas y económicas de los participantes.

Este trabajo constituye un esfuerzo para desarrollar y validar un instrumento basado en el modelo teórico de bienestar eudemónico pero diseñado ex profeso para adolescentes mexicanos. Los resultados encontrados confirman su pertinencia y la necesidad de considerar las influencias culturales al medir constructos psicológicos. La evidencia acumulada hasta el momento confirma la mutidimensionalidad del constructo de BP, pero quedan muchas interrogantes respecto a qué y cuántas dimensiones lo conforman y cómo cambian a lo largo de las diversas etapas que conforman el ciclo de vida en diversos contextos sociales y culturales.

\section{Referencias}

Abbott, R., Ploubidis, R., Hupper, F., Kuh, D., Wadsworth, M., \& Croudace, T. (2006). Psychometric evaluation and predictive validity of Ryff's psychological well-being items in a UK birth cohort sample women. Health and Quality of Life Outcomes, 4: 76. doi: 10.1186/1477-5225-4-76

Akin, A. (2008). The scales of psychological wellbeing: A study of validity and reliability. Educational Science: Theory and Practice, 8(3), 741-750.

Balcázar, N. P, Bonilla, M. M., Gurrola, P. G., Loera, M., \& Trejo, G. L. (2009). Adaptación de la escala de bienestar psicológico de Ryff en adolescentes preuniversitarios. Revista Científica Electrónica de Psicología, 7, 69-86. Universidad Autónoma del Estado de México. Biswas-Diener, R., Kashdan, T., \& King, L. (2009). Two traditions of happiness research, not two distinct types of happiness. The Journal of Positive Psychology, 4(3), 208211.

Bradburn, N. (1969). The structure of psychological well-being. Chicago: Aldine Pub. Co., 1969. ONLINE ED: Published May 2001 by the NORC Library. Recuperado en: http://cloud9.norc.uchicago.edu/dlib/spwb/ind ex.htm

Bronk, K. C., Finch, W.H., \& Talib, L.T. (2010). Purpose in life among high ability adolescents. High Ability Studies, 21(2), 133145.

Bronk, K. C., Hill, P., Lapsley. D.K., Talib, T., \& Finch, H. (2009). Purpose, hope, and life satisfaction in three age groups. The Journal of Positive Psychology, 4(6), 500-510.

Burns, R., \& Machin, A. (2009). Investigating the structural validity of Ryff'psychological wellbeing scales across two samples. Social Indicators Research, 93(2), 359-366.

Casullo, M. M. (2002). Evaluación del bienestar psicológico. En M. Casullo, M.E. Brenlla, A. 
Castro S., A., Cruz, R. González, C. Maganto, M. Martín, I. Montoya, \& R. Morote. Evaluación del bienestar psicológico en Iberoamérica (pp.11-29). Buenos Aires: Paidós.

Casullo, M. M., Brenlla, M.E., Castro, S. A., Cruz, A., González, R., Maganto, C., Martín, M., Montoya I., \& Morote, R. (2002). Evaluación del bienestar psicológico en Iberoamérica. Buenos Aires: Paidós.

Casullo, M. M., \& Castro Solano, A. (2000). Evaluación del bienestar psicológico en estudiantes adolescentes argentinos. Revista de Psicología de la Pontificia Universidad Católica de Perú, 18(1), 36-68.

Casullo, M. M., \& Castro Solano, A. (2001). El significado de bienestar en estudiantes adolescentes. Revista Iberoamericana de Diagnóstico y Evaluación - e Avaliação Psicológica, 12(2), 57-70.

Cheng, S., \& Chan, A. (2005). Measuring psychological well-being in Chinese. Personality and Individual Differences, 38, 1307-1316.

Clarke, P., Marshall, V., Ryff, C., \& Wheaton, W. (2001). Measuring psychological well-being in the Canadian study of health and aging. International Psychogeriatrics, 13(1), 79-90.

Contini de González, E. N., Coronel de Pace, C., Levin, M., \& Esteves Suedan, M. A. (2003). Estrategias de afrontamiento, bienestar psicológico y factores de protección de la salud del adolescente. Un estudio preliminar. Revista Iberoamericana de Diagnóstico y Evaluación - e Avaliação Psicológica, 16(2), 29-51.

Cruz, M.S., Maganto, C. Montoya, I., \& González R. (2002). Escala de bienestar psicológico para adolescentes. En M. Casullo, M.E. Brenlla, A. Castro S., M. Cruz, R. González, C. Maganto, M. Martín, I. Montoya, \& R. Morote. Evaluación del bienestar psicológico en Iberoamérica. (pp.65-77). Buenos Aires: Paidós.

Deci, E.L., \& Ryan, R. M. (1985). Intrinsic motivation and self-determination in human behavior. New York: Plenum.

Deci, E.L. \& Ryan, R. M. (2000). The "What" and "Why" of goal pursuits: Human needs and the
Self-determination of behavior. Psychological Inquiry, 11(4), 227-268 Doi: 10.1207/S15327965PLI1104_01

Deci, E. L. \& Ryan, R. M. (2008). SelfDetermination Theory: A macrotheory of human motivation, development, and health. Canadian Psychology, 49(3), 182-185.

Delle Fave, A., \& Bassi, M. (2009). The contribution of diversity to happiness research. Journal of Positive Psychology, 4(3), 205-207.

Díaz, D., Rodríguez-Carbajal, R., Blanco, A., Moreno-Jiménez, B., Gallardo I., Valle, C., \& van Dierendonck, D. (2006). Adaptación española de las escalas de bienestar psicológico de Ryff. Psicothema, 18, 572-577.

Diener, E. (1984). Subjective well-being. Psychological Bulletin, 95, 542-575.

Diener, E., Emmons, R., Larsen, R., \& Griffin, S. (1985). The satisfaction with life scale. Journal of Personality Assessment, 49, 71-75.

Diener, E., Suh, E., Lucas, R., \& Smith, H. (1999). Subjective well-being: Three decades of progress. Psychological Bulletin, 125, 276302.

Kafka, G., \& Kozma, A. (2002). The construct validity of Ryff's scales of psychological well-being (SPWB) and their relationship to measures of subjective well-being. Social Indicators Research, 57, 171-190

Kahneman, D., Diener, E., \& Schwarz, N. (1999). (Eds.) The foundations of hedonic Psychology. USA Russell Sage Foundation.

Kashdan, T., Biswas-Diener, \& King, L. (2008) Reconsidering happiness: The cost of distinguishing between hedonic and eudaimonia. The Journal of Positive Psychology, 3(4), 219-233.

Keyes, C. L. M., \& Annas, J. (2009). Feeling good and functioning well: Distinctive concepts in ancient philosophy and contemporary science. The Journal of Positive Psychology. 4(3), 197-201. doi: 10.1080/17439760902844228

Keyes, C. L. M., Shmotkin, D., \& Ryff, C. D. (2002). Optimizing well-being: The empirical encounter of two traditions. Journal of Personality and Social Psychology. 82, 10071022 
Krueger, R., \& Casey, M.A. (2000). Focus groups. A practical guide for applied research. USA: Sage Publications, Inc.

Kvale, S. (2007). Doing Interviews. London: Sage Publications, Ltd.

Lindfors, P., Berntsson, L., \& Lundberg, U. (2006). Factor structure of Ryff's psychological well-being scales in Swedish female and male white-collar workers. Personality and Individual Differences, 40, 1213-1222.

Martínez, P., \& Morote, R. (2002). El bienestar psicológico en adolescentes escolares de Lima Metropolitana. En: M. Casullo, M.E. Brenlla, A. Castro S., M. Cruz, R. González, C. Maganto, M. Martín, I. Montoya \& R. Morote. Evaluación del bienestar psicológico en Iberoamérica.(pp. 55-64). Buenos Aires: Paidós.

Medina-Calvillo, M., Gutiérrez-Hernández, C., \& Padrós-Blázquez, F. (2013). Propiedades psicométricas de la escala de bienestar psicológico de Ryff en población mexicana. Revista de Educación y Desarrollo, 27, Octubre-diciembre, 25-31.

Ryan, R., \& Deci, E. (2000). Self-Determination theory and the facilitation of instrinsic motivation, social development, and wellbeing. American Psychologist, 55(1), 68-78. doi: 10.1037//0003-066X.55.1.68

Ryan, R., \& Deci, E. (2001). On happiness and human potentials: A review of research on hedonic and eudaimonic well-being. Annual Review Psychology, 52, 141-166.

Ryan, R., \& Huta, V. (2009). Wellness as healthy functioning or wellness as happiness: The importance of eudaimonic thinking (response to Kashdan et al. and Waterman discussion). Journal of Positive Psychology, 4(3), 202204.

Ryff, C. (1989a). Beyond Ponce de Leon and life satisfaction: New directions in quest of successful ageing. International Journal of Behavioral Development, 12(1), 35-55.

Ryff, C. (1989b). Happiness is everything, or is it? Explorations on the meaning of psychological well-being. Journal of Personality and Social Psychology, 57, 1069-1081.
Ryff, C. (1995). Psychological Well-Being in adult life. Current Directions in Psychological Science, 4(4), 99-104. Recuperado de http:/www.jstor.org/stable/20182342

Ryff, C., \& Heidrich, S. (1997). Experience and well-being: Explorations on domains of life and how the matter. International Journal of Behavioral Development, 20(2), 193-206.

Ryff, C., \& Keyes, C. (1995). The structure of psychological well-being revisited. Journal of Personality and Social Psychology, 69, 719727.

Ryff, C., \& Singer, B. (1998). The contours of positive human health. Psychological Inquiry, 9, 1-28.

Ryff, C., \& Singer, B. (2006). Best news yet on the six-factor model of well-being. Social Science Research, 35, 1103-1119.

Ryff, C., \& Singer, B. (2008). Know thyself and become what you are: A Eudaimonic approach to psychological well-being. Journal of Happiness Studies, 9, 13-39. doi: 10.007/s10902-006-9019-0.

Ryff, C., Singer, B., \& Dienberg, G. (2004) Positive health: Connecting well-being with biology. Phil. Trans.R.Soc.Lond., 359, 13831394. Published on line 11 august, 2004 The Royal Society. Recuperado de: http://rstb.royalsocietypublishing.org/

Schmute, P., \& Ryff, C. (1997). Personality and well-being: Reexamining methods and meanings. Journal of Personality and Social Psychology, 73(3), 549-559.

Springer, K. W., \& Hauser, R. M. (2006). An assessment of the construct validity of Ryff's scales of psychological well-being: Method, mode, and measurement effects. Social Science Research, 35, 1079-1101.

Springer, K. W., Hauser, R. M., \& Freese, J. (2006). Bad news indeed for Ryff's six factor model of well-being. Social Science Research, 35, 1120-1131.

Tomás, J. M., Meléndez, J. C. y Navarro, E. (2008). Modelos factoriales confirmatorios de las escalas de Ryff en una muestra de personas mayores. Psicothema, 20(2), 304310. www.psicothema.com

Triadó, C., Villar, F., Solé, C., \& Celdrán, M. (2007). Construct validity of Ryff's scale of 
psychological well-being in Spanish older adults. Psychological Reports, 100, 11511164. doi: 10.2466/PRO.100.4.1151-1164

Van Dierendonck, D. (2005). The construct validity of Ryff's scales of psychological well-being and its extension with spiritual well-being. Personality and Individual Differences, 36(3) 629-643.

Van Dierendonck, D., Díaz, D., RodríguezCarvajal, R., Blanco, A., \& Moreno Jiménez, B. (2008). Ryff's six factors model of psychological well-being, a spanish exploration. Social Indicators Research, 87, 473-479.

Vleioras, G., \& Bosma, H. (2005). Are identity important for psychological well-being? Journal of Adolescence, 28, 397-409. doi:10.1016/j.adolescence.2004.09.001

Waterman, S.A. (1993). Two conceptions of happiness: Contrasts of personal expressiveness (eudemonia) and hedonic enjoyment. Journal of Personality and Social Psychology, 64(4), 678-691.

Waterman, S. A. (2008). Reconsidering happiness: A eudaemonist's perspective. Journal of Positive Psychology, 3, 234-252.

Waterman, S. A., Schwartz, S., Zamboanga, B., Ravert, R., Williams, M., Agocha, V. ..., \& Donnellan, M.B. (2013). The questionnaire for eudamonic well-being: Psychometric properties, demographic comparison, and evidence of validity. Journal of Positive Psychology, 5(1), 41-61. 